phys. stat. sol.

\title{
Influence of Dopants on Defect Formation in GaN
}

\author{
Z. LILIENTAL-WEBER ${ }^{1}$ (a), J. JASINSKI (a), M. BENAMARA (a) ${ }^{2}$, I. \\ GRZEGORY (b), S. POROWSKI (b), D.J. H. LAMPERT (c), C.J. EITING (c), \\ and R.D. DUPUIS (c) \\ (a) Lawrence Berkeley National Laboratory, Berkeley, CA 94720; USA, m/s \\ $62 / 203$ \\ (b) High Pressure Research Center "Unipress, ” Polish Academy of Sciences, \\ Warsaw, Poland \\ (c) University of Texas at Austin, Microelectronics Research Center, Austin TX \\ 78712, USA
}

(Submitted July 14, 2001)

Subject classification:68.37Lp; S7.14

Influence of p-dopants ( $\mathrm{Mg}$ and $\mathrm{Be}$ ) on the structure of $\mathrm{GaN}$ has been studied using Transmission Electron Microscopy (TEM). Bulk GaN:Mg and GaN:Be crystals grown by a high pressure and high temperature process and $\mathrm{GaN}: \mathrm{Mg}$ grown by metal-organic chemical-vapor deposition (MOCVD) have been studied. Structural dependence on growth polarity was observed in the bulk crystals. Spontaneous ordering in bulk GaN:Mg on cplane (formation of Mg-rich planar defects with characteristics of inversion domains) was observed for growth in the $\mathrm{N}$ to Ga polar direction ( $\mathrm{N}$ polarity). On the opposite site of the crystal (growth in the $\mathrm{Ga}$ to $\mathrm{N}$ polar direction) $\mathrm{Mg}$-rich pyramidal defects empty inside (pinholes) were observed. Both these defects were also observed in MOCVD grown crystals. Pyramidal defects were also observed in the bulk GaN:Be crystals.

\section{Introduction}

$\mathrm{GaN}$ can easily be grown with n-conductivity but obtaining p-doping is rather difficult. Only recently p-doping was also obtained by Be doping [1]. Mg is most commonly used as the p-dopant in GaN; however, higher hole concentrations can only be obtained after thermal annealing [2] in order to dissociate $\mathrm{Mg}-\mathrm{H}$ complexes. Material made using this process has been used to fabricate light emitting diodes (LEDs) [3] and lasers [4]. Despite this success, many aspects of Mg-doping in GaN are still not fully understood. Transmission electron microscopy (TEM) studies show formation of different types of $\mathrm{Mg}$ rich defects in many bulk crystals. The type of defects formed depends strongly on the growth polarity. In the bulk GaN:Mg platelet crystals, planar defects distributed in equal distances (20 unit cells of $\mathrm{GaN}$ ) leading to superlattice reflections in the diffraction pattern can be observed [5-7] for growth with N-polarity. Similar defects are also formed in layers grown by metal-organic chemical-vapor deposition (MOCVD) with Mg delta doping [6-7]. For majority of platelet crystals grown with Ga polarity, formation of pyramidal defects empty inside but rich in $\mathrm{Mg}$ on their walls was observed. These pyramidal defects are also typical for all MOCVD-grown crystals, and their high concentration can be observed in all crystals for a wide range of $\mathrm{Mg}$ concentration.

1) Corresponding author : phone 510-486-6276, Fax 510-486-4995, e-mail:

2)_z_liliental-weber@lbl.gov; 2) at present-Max Planck Institute, Halle, Germany 
phys. stat. sol.

Bulk GaN:Be crystals are somehow similar to GaN:Mg. As-grown surface is rough for growth with N-polarity. However, spontaneous ordering was not observed. However, for growth with Ga polarity, pyramidal defects are also observed in some crystals.

\section{Experimental}

Different types of GaN crystals doped by $\mathrm{Mg}$ and some with Be have been studied using plan-view and cross-sectioned samples. Bulk crystals were grown by the High Nitrogen Pressure Method [8] from a solution of liquid gallium containing $0.1-0.5$ at.\% Mg [9]. Similar procedure was applied to GaN:Be crystals. Two types of samples were grown using MOCVD where $\mathrm{Mg}$ was added either continuously during the growth or by the delta doping method (one monolayer at each $100 \AA$ of $\mathrm{GaN}$ ). The details of this growth have been described earlier [6-7]. Following the growth, the temperature was lowered to $850^{\circ} \mathrm{C}$, the ambient was switched to nitrogen only, and a 10 minute in-situ anneal was performed to dissociate the Mg-H complexes and activate the $\mathrm{Mg}$ atoms [10]. The same growth temperature and post annealing was applied to the crystals for which $\mathrm{Mg}$ was added continuously during growth. All GaN:Mg and the GaN:Be crystals have been studied using TEM on plan-view and cross-section samples, transparent for electrons, prepared by standard methods using Topcon 002B and JEOL ARM with the accelerating voltages of 200 and $800 \mathrm{kV}$, respectively.

\section{Results}

A strong dependence of structure on crystal growth polarity was observed in bulk platelet crystals. For the crystal side grown with $\mathrm{N}$ polarity ( $\mathrm{N}$ to Ga direction) many crystals were free of defects, but Mg-rich planar defects were observed in some crystals, despite the fact that $\mathrm{Mg}$ concentrations determined by SIMS remain practically unchanged. These defects
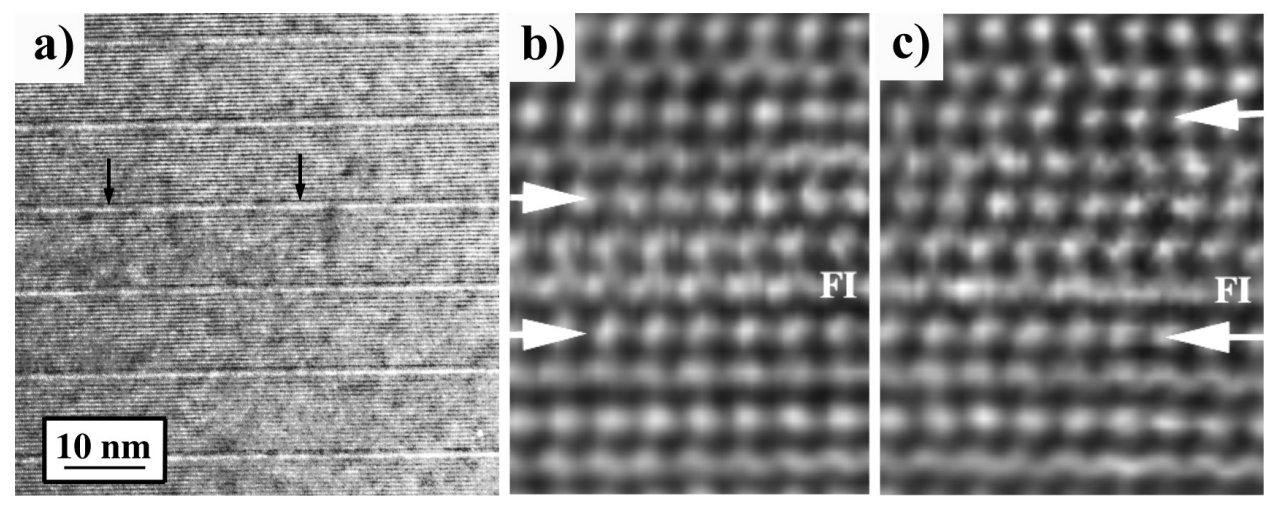

Fig.1: (a) TEM micrograph showing equi-distant arrangement of planar defects (inversion domain-ID) formed in bulk GaN crystal grown with N-polarity. Arrows indicate areas from which higher magnification micrographs are shown in (b) and (c), respectively. The white arrows in (b) and (c) indicate the position of perfect $\mathrm{GaN}$ matrix on both side of the planar defect. FI is a flat interface of the inversion boundary which remains on the same c-plane, but the upper interface, called corrugated interface (CI) is changing from place to place. 
phys. stat. sol

can be perfectly ordered for several micrometers from the sample surface. They can form a perfect array of planar defects separated from each other by 20 unit cells of GaN [5-7] (Fig. 1a). Perfect ordering leads to super-lattice spots dividing the (0001) lattice distance into 20 equal parts (not shown here). The following TEM results, e.g. presence of split (0001) reflection, reverse contrast for multi-beam dark-field (0001) and (000-1) reflections, symmetrical contrast in dark field (top and bottom surface of TEM foil) indicate that these planar defects are inversion domains [5-7] formed on c-planes with a very narrow width along the c-axis. In addition, it was observed that pairs of inversion boundaries in one domain are separated by not more than 1-3 unit cells. One of the domain boundaries called the flat interface (FI) shown in Figs (1b and c) always remains on the same plane. The upper boundary, called the corrugated interface, changes its position, so that the domain wall thickness is changing from $3 / 2 \mathrm{c}$ to $5 / 2 \mathrm{c}$ as shown by high resolution images taken in two areas from the same defects (Figs. $1 \mathrm{~b}$ and $\mathrm{c}$ ). The distribution of these inversion domains is shown graphically on Fig. 2. Local change of thickness within one domain gives additional evidence that the observed planar defects are not stacking faults. EDX studies gave evidence of $\mathrm{Mg}$ enhancement within the domains [5]. This perfect ordering was not observed in all crystals studied. In some crystals this perfect ordering was interrupted by larger areas of hexagonal $\mathrm{GaN}$ or this ordering can be interrupted by stacking fault.

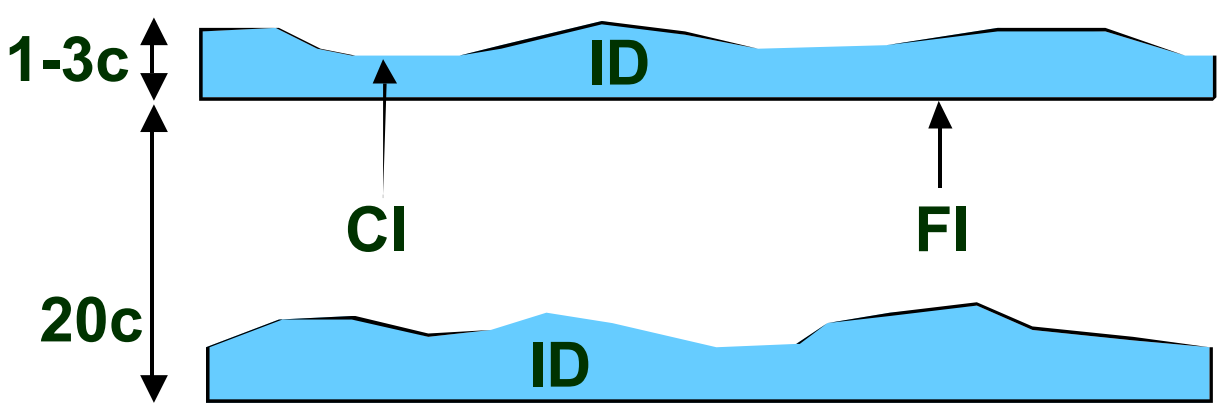

Figure 2: Schematic showing distribution of inversion domains (ID) in GaN grown with $\mathrm{N}$-polarity. The thickness of particular domains is changing locally but distance between equivalent domains remains $20 \mathrm{c}$ (unit cells).

The opposite side of the bulk crystals (grown with Ga-polarity) had a completely different defect structure as shown in Fig. 3 (a). They appear in [11-20] cross-section TEM micrographs as triangular features with a base on the (0001) c-plane and six side facets inclined $45^{\circ}$ to the basal plane. The dimension of the largest defects varies. The longest base length of these defects are in the range $100 \mathrm{~nm}$ and the smallest about 3-5 nm. The density of these defects is in the range $2.5 \times 10^{9} \mathrm{~cm}^{-2}$. All these triangles were oriented in a direction with the base closest and parallel to the sample surface with Ga-polarity, e.g. from the triangle tip to the base, the long bond direction along the c-axis is from Ga to N. Study of these defects in plan-view configuration prove that these defects are empty inside (Fig. 3b). EDX studies showed that $\mathrm{Mg}$ is segregated on the base and side-walls of these defects. High resolution TEM images from the defect base show the formation of planar defect. 
phys. stat. sol.
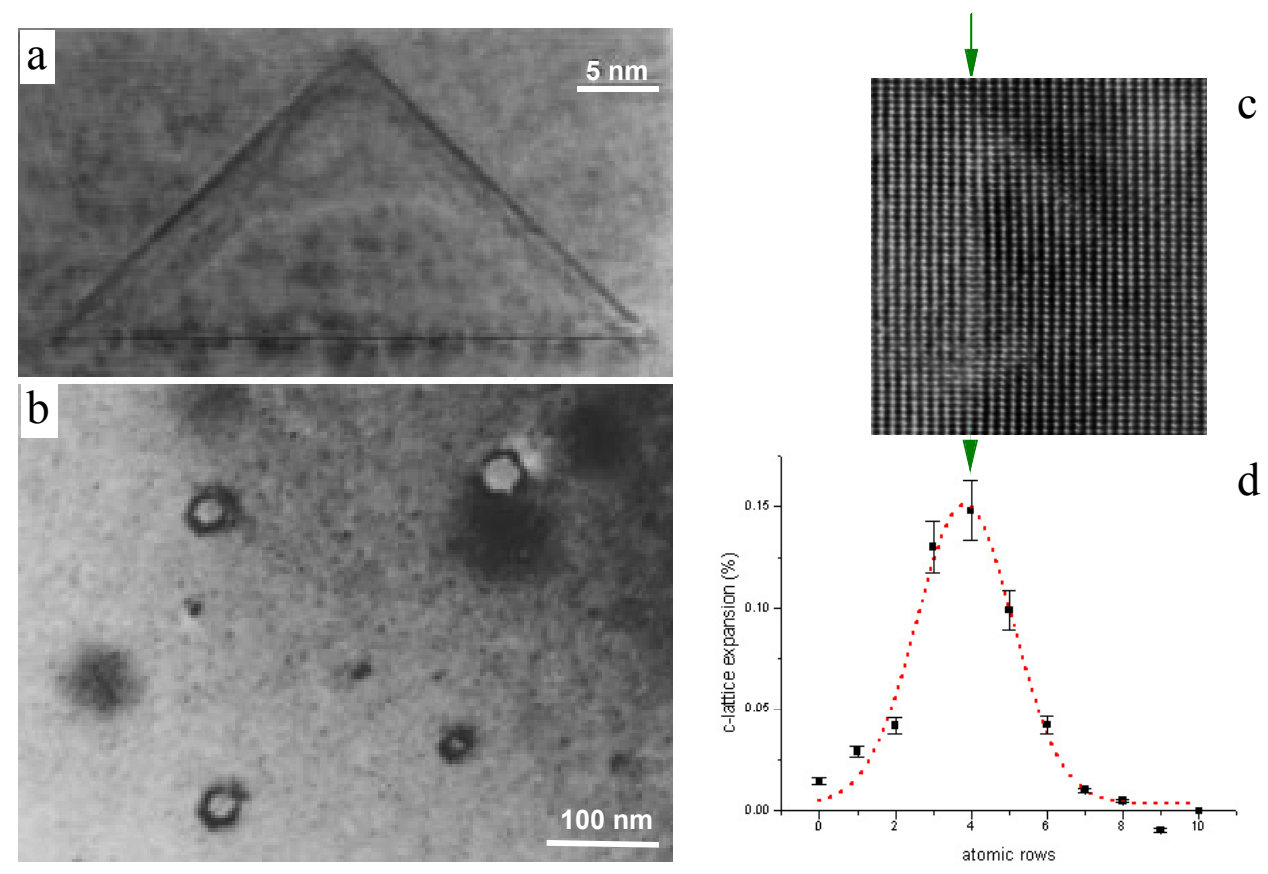

Fig. 3. (a) TEM cross-section micrographs showing defects formed in platelet $\mathrm{GaN}: \mathrm{Mg}$ bulk crystals on the side grown with Ga polarity observed in cross-section, (b) the same defects observed in plan-view configuration; (c) high resolution micrograph observed in MOCVD grown crystals showing expansion of the interplanar distances in the defect base; (d) measured lattice expansion in the defect base.

\section{GaN:Mg layers grown by MOCVD}

Since the majority of layers applied in devices are grown by MOCVD it was also interesting to observe the structure of p-doped layers to learn why p-doping is so difficult [2]. Two types of crystals have been studied: one where $\mathrm{Mg}$ was added as delta doping, to study if addition of a monolayer of $\mathrm{Mg}$ at equal distances will lead to the ordering observed in bulk crystals and the second where $\mathrm{Mg}$ was added continuously, as used for growth in devices.

Cross-section TEM studies of Mg-delta doped samples show both types of defects observed for the two opposite growth polarities in bulk GaN:Mg, e.g. planar defects observed earlier for $\mathrm{N}$ growth polarity and pyramidal defects observed in the bulk GaN:Mg for growth with Ga polarity [5-7]. In a crystal with Mg-delta doping, planar defects were formed followed by a high density of pyramidal defects. In this crystal a layer about 100 $\mathrm{nm}$ thick with planar defects (Fig. 4a), like those observed in bulk crystals grown with Npolarity, was observed at about $150 \mathrm{~nm}$ distance from the buffer layer. This suggest that some accumulation of $\mathrm{Mg}$ on the surface is needed for the first Mg-rich planar defect to appear. Further growth resulted in a random distribution of pyramidal defects with a density of $\sim 10^{10} \mathrm{~cm}^{-2}$. High -resolution TEM images from the defect base show an expansion of the 
phys. stat. sol

interplanar distance (Fig. 3c): 14\% expansion was measured (Fig. 3d). This value was compared to the expansion expected if $\mathrm{Mg}, \mathrm{MgO}, \mathrm{MgO}_{2}$, or predicted theoretically $\mathrm{Mg}_{3} \mathrm{~N}_{2}$ phase would be formed on the defect base. The best agreement was obtained for $\mathrm{Mg}$ accumulating on the $\mathrm{c}$ planes in the base of these defects (Table 1).

Table 1: Calculated expansion of interplanar distances in the pyramidal defect base for different materials

\begin{tabular}{|c|c|c|}
\hline \multirow{2}{*}{} & \multicolumn{2}{|c|}{$\mathrm{GaN}$} \\
\cline { 2 - 3 } & $\mathrm{c} / 2=2.585 \AA$ & $\mathrm{a}=3.18 \AA$ \\
$\mathrm{MgO} / 2=2.585 \AA$ & $18 \%$ & \\
\hline $\begin{array}{c}\mathrm{MgO}_{2} \\
\mathrm{a} / 2=2.422 \AA\end{array}$ & $6 \%$ & \\
\hline $\begin{array}{c}\mathrm{Mg} \\
\mathrm{a}=2.95 \AA\end{array}$ & $14 \%$ & $7 \%$ \\
\hline $\begin{array}{c}\mathrm{Mg}_{3} \mathrm{~N}_{2} \\
\mathrm{a} / 4=2.45 \AA\end{array}$ & $4 \%$ & \\
\hline
\end{tabular}
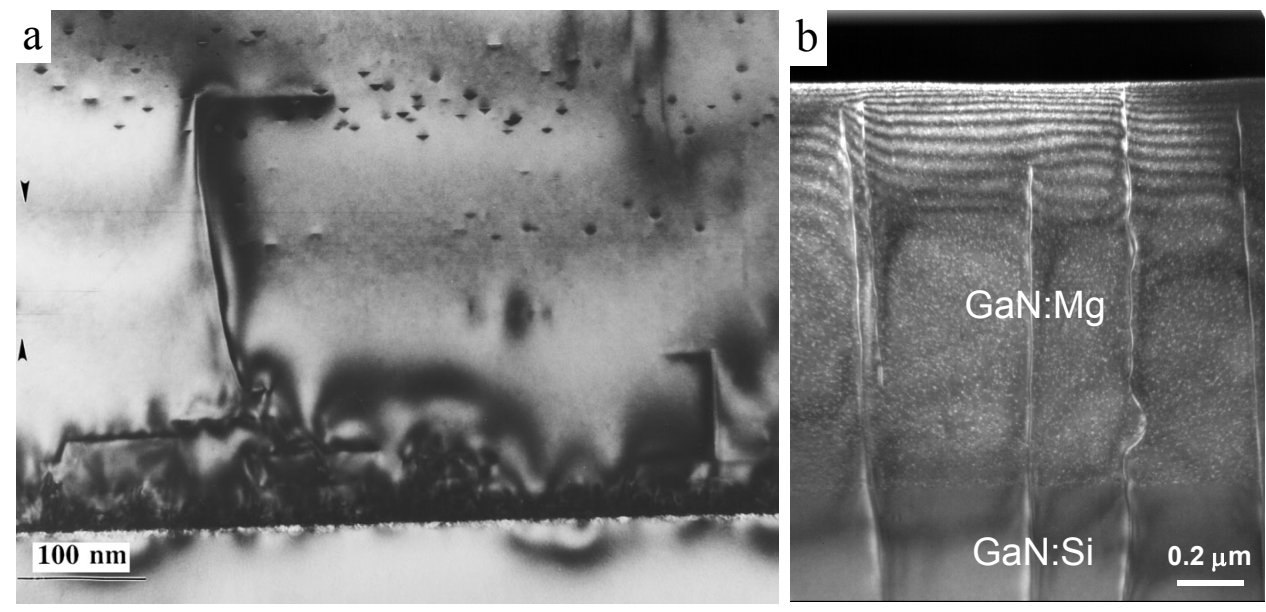

Fig. 4 (a) Cross-section TEM micrographs showing a layer with delta doping grown with $\mathrm{GaN}$ buffer layer on sapphire. The area between the arrows shows planar defects similar to these observed in bulk platelet crystals grown with N-polarity. These planar defects delay to appear compared to the expected location of the first monolayers of $\mathrm{Mg}$. Above the top arrow, $\mathrm{Mg}$ monolayers were not found, but pyramidal defects (same as in bulk crystals grown with Ga polarity) in high density were observed. Some of these pyramidal defects started to appear simultaneously with the planar defects.(b) A layer of $\mathrm{GaN}: \mathrm{Mg}$ with continuous Mg doping. Note high density of pyramidal defects (white dots). 
phys. sta. sol.

SIMS analysis in the layers with $\mathrm{Mg}$ delta doping indicated that $\mathrm{Mg}$ concentration was steadily increasing and it reached the level of $4 \times 10^{19} \mathrm{~cm}^{-3}$ in the area with planar defects [7]. In the area where the hollow defects (rectangular and triangular) were formed $\mathrm{Mg}$ concentration dropped to $2 \times 10^{19}$ atoms $/ \mathrm{cm}^{3}$ and stayed almost constant with a slow increase to reach a concentration of $4 \times 10^{19}$ at the sample surface[7].

In the samples where $\mathrm{Mg}$ was added continuously (with comparable $\mathrm{Mg}$ concentration to that in the previous samples), no planar defects were observed but a high density of pyramidal defects was present (Fig. 4b). These pyramids are also empty inside but their size was much smaller compared to these observed in bulk platelets ( $8 \mathrm{~nm}$ in the base). At the base of these defects stacking faults were not formed as in the larger pyramids observed in the bulk samples. However, the expansion of the lattice parameter of about $14 \%$ was observed in two basal parallel layers (Fig. 3c and d). The expansion agrees well with what would be expected if $\mathrm{Ga}$ atoms were substituted by $\mathrm{Mg}$.

Table 2. SIMS analysis of bulk platelets for growth with $\mathrm{N}$ - and Ga-polarity where either ordering (ord) or pyramidal defects (def) were observed and defect free samples (no ord. and no def) compared to MOCVD samples grown with Mg delta ( $\delta$ ) doping and continuous (c) Mg doping. Concentralion for particular elements $\left(\mathrm{cm}^{-3}\right)$.

\begin{tabular}{|c|c|c|c|c|}
\hline Sample & $\mathrm{Mg}$ & $\mathrm{O}$ & $\mathrm{C}$ & $\mathrm{Si}$ \\
\hline $\begin{array}{c}\text { A } \\
\text { Ga-def }\end{array}$ & $\begin{array}{c}\mathrm{e} 20-6 \mathrm{e} 19 \\
6 \mathrm{e} 19\end{array}$ & $2.5 \mathrm{e} 19$ & $1.5 \mathrm{e} 17$ & $4 \mathrm{e} 16$ \\
\hline $\begin{array}{c}\text { B } \\
\text { N-par.ord } \\
\text { Ga-no def }\end{array}$ & $5 \mathrm{e} 19$ & $3 \mathrm{e} 19$ & $5 \mathrm{e} 19$ & $1 \mathrm{e} 16-1 \mathrm{e} 17$ \\
\hline $\begin{array}{c}\mathrm{C} \\
\text { N-no ord. } \\
\text { Ga-def }\end{array}$ & $4-7 \mathrm{e} 19$ & $2 \mathrm{e} 19$ & $3 \mathrm{e} 17$ & $1-3 \mathrm{e} 17$ \\
\hline $\begin{array}{c}\mathrm{D} \\
\text { N-no ord. } \\
\text { Ga-no def. }\end{array}$ & $4-6 \mathrm{e} 19$ & $7 \mathrm{e} 19$ & $1.5 \mathrm{e} 18$ & $1-2 \mathrm{e} 19$ \\
\hline $\begin{array}{c}\text { MOCVD- } \delta \\
\text { ord.+def. }\end{array}$ & $1.5-3 \mathrm{e} 19$ & $5 \mathrm{e} 16$ & $5 \mathrm{e} 16$ & ---- \\
\hline $\begin{array}{c}\text { MOCVD-c } \\
\text { def }\end{array}$ & $3 \mathrm{e} 19$ & $5 \mathrm{e} 16$ & $5 \mathrm{e} 16$ & \\
\hline
\end{tabular}

\section{Discussion of Mg results}

TEM studies of bulk crystals grown under high pressure and high temperature doped with $\mathrm{Mg}$ and those grown by MOCVD show formation of structural defects for a wide range of $\mathrm{Mg}$ concentration. This would explain (at least to some extend) why it is so difficult to obtain high hole concentration despite nominal higher $\mathrm{Mg}$ concentration in the crystal. Since p-conductivity was not obtained in bulk crystals, but GaN:Mg samples became semi-insulating, some compensation of oxygen presence in these samples took place. Ordering of planar defects observed in GaN:Mg formed for growth with N-polarity 
phys. stat. sol.

appears to be similar to the polytypoids formed in AlN rich in oxygen [11]. But it appears that similar defects were formed in MOCVD grown samples, where oxygen concentration was three orders of magnitude lower. Therefore, it is believed that mainly Mg is responsible for the formation of these defects. Our studies of bulk samples from two opposite sides, e.g. grown with $\mathrm{N}$ and Ga-polarity, do not indicate substantial difference in $\mathrm{Mg}$ incorporation for different growth polarity as predicted theoretically [12] and observed in MBE grown samples [13], where up to 30 times more $\mathrm{Mg}$ was incorporated in Ga-polarity, as determined by secondary ion mass spectrometry. The last authors [13] also indicated accumulation of $\mathrm{Mg}$ on the sample surface which would be consistent with our results.

\section{GaN:Be bulk samples}

TEM studies of Be-doped GaN platelet crystals show many similarities to the samples grown by the same method with Mg. However, ordering was not observed in these samples. For growth with N-polarity as-grown crystals appear to have rough surface (Fig. 5a) and the growth with Ga polarity results in smooth surface (Fig. 5b). Only three crystals have been studied. Two of them did not show any structural defects, but pyramidal defects have been observed in one of them Figs. ( $5 \mathrm{c}$ and d). The size of these pyramids appear much smaller in comparison to those observed in $\mathrm{Mg}$ doped platelet samples. The length of the base of these defects is in the range of $5-10 \mathrm{~nm}$, similarly to the sizes observed in $\mathrm{GaN}: \mathrm{Mg}$ grown by MOCVD. The density of these defects appears also lower in comparison to Mg doped crystals. These samples are also semi-insulating.
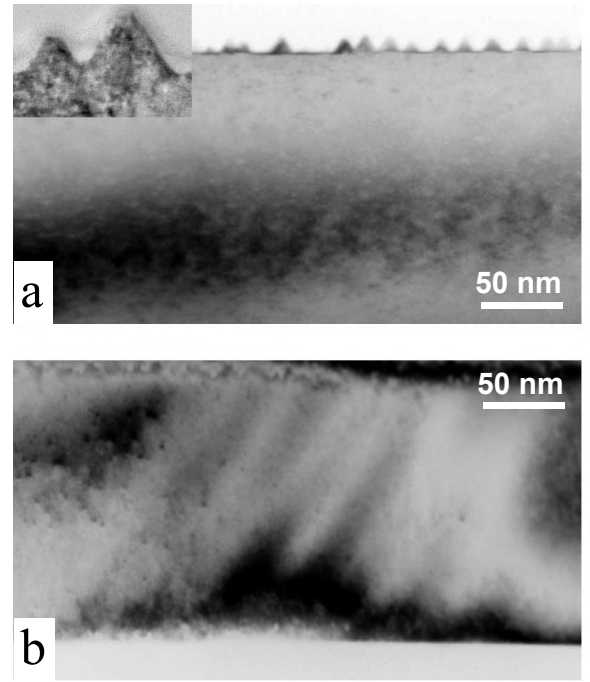
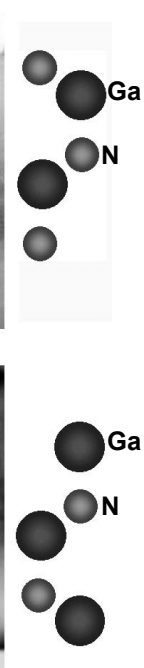
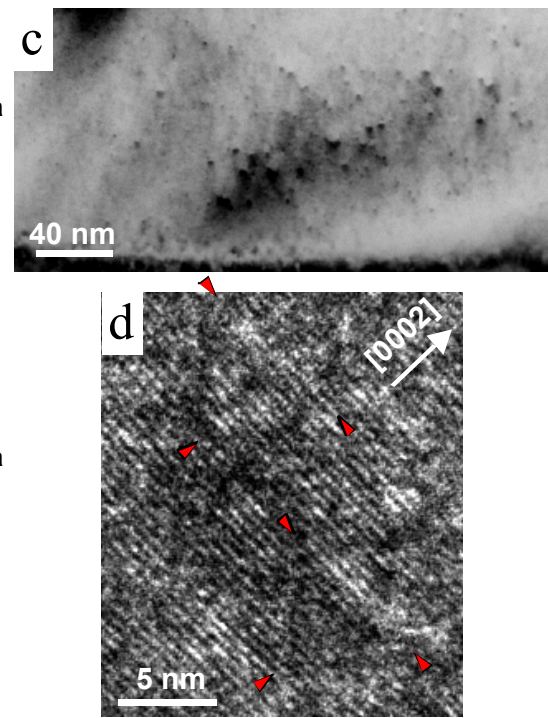

Fig. 5: (a) TEM micrograph of GaN:Be platelet with corrugated surface. (b) The same sample from the opposite side showing flat surface. (c) Part of another platelet crystal grown with Ga polarity with pyramidal defects (small dots). (d) High resolution image of two pyramids which corners were indicated by arrows. 
phys. stat. sol.

\section{Summary}

TEM studies of Mg-doped GaN crystals show formation of structural defects for a wide range of Mg concentration strongly influenced by crystal growth polarity. Similar defects are formed for bulk crystals grown under high hydrostatic pressure and for those grown by MOCVD. In some bulk crystals for the plate side grown with N-polarity equally distributed planar defects were observed (20 unit cells). Each defect appears to be an inversion domain with domain thickness about 1-3 unit cells along c-axis. No inversion in the GaN between these planar defects was observed.

Pyramidal defects, were observed for growth with $\mathrm{Ga}$ polarity, also due to $\mathrm{Mg}$ segregation. These defects were observed in the majority of bulk GaN samples and in all samples grown by MOCVD. When these pyramids are small, a 14\% lattice expansion can be detected in the bases of the pyramids consistent with the substitution of $\mathrm{Ga}$ by $\mathrm{Mg}$ atoms.

The samples grown with Be doping show similarities with $\mathrm{Mg}$ - doped samples in respect to their surface roughness and formation of pyramidal defects in some crystals. Planar defects were not observed in these crystals. Semi-insulating properties were measured in the crystals doped with $\mathrm{Mg}$ and $\mathrm{Be}$.

\section{Acknowledgment}

This work was supported by the U.S. Department of Energy, under the Contract No. DEAC03-76SF00098.

\section{References}

1.T.H. Myers, .A.J. Ptak, ; L. Wang, .N.C. Giles, . Proc. Int. Workshop on Nitride Semiconductors, Nagoya, Japan, 24-27 Sept. (2000).

2. H. Amano, M. Kito, K. Hiramatsu, and I. Akasaki, Inst. Phys. Conf. Ser. 106, 725 (1989). 3. S. Nakamura, M. Senoh, and T. Mukai, Jpn. J. Appl. Phys. 31, L1708 (1991).

4. S. Nakamura, Paper Plenary 1, presented at the 24th International Symposium on Compoud Semiconductors, San Diego CA, 8-11 September (1997).

5.Z. Liliental-Weber, M. Benamara, J. Washburn, I. Grzegory, and S. Porowski, Phys. Rev. Lett. 83, 2370 (1999).

6.Z. Liliental-Weber, M. Benamara, W. Swider, J. Washburn, I. Grzegory, S. Porowski, D.J.H. Lambert, C.J. Eiting, and R.D. Dupuis, Appl. Phys. Lett. 75, 4159 (1999).

7.Z. Liliental-Weber, M. Benamara, W. Swider, J. Washburn, I. Grzegory, S. Porowski, D.J.H. Lambert, C.J. Eiting, and R.D. Dupuis, Physica B 273-274, 124 (1999).

8. I. Grzegory, J. Jun, M. Bockowski, S. Krukowski, M. Wroblewski, B. Lucznik, and S. Porowski, J. Phys. Chem. Solids 56, 639 (1995).

9. S. Porowski, M. Bockowski, B .Lucznik, I. Grzegory, M. Wroblewski, H. Teisseyre, M. Leszczynski, E. Litwin-Staszewska, T .Suski, P. Trautman, K. Pakula and J.M. Baranowski, Acta Physica Polonica A 92, 958 (1997).

10.C.J. Eiting, P.A. Grudowski, J.S. Park, D.J.H. Lambert, B.S. Shelton, and R.D. Dupuis, J. Electrochem. Soc. 144, L219 (1997).

11. R.A. Youngman, A.D. Westwood, and M.R. McCartney, Mat. Res. Soc. Symp. Proc. 319, 45 (1994).

12. C. Bungaro, K. Rapcewicz, and J. Bernholc, Phys. Rev. B59, 9771 (1999).

13. A. J. Ptak and T. H. Myers, L. T. Romano, C. G. Van de Walle, and J. E.Northrup, Appl. Phys. Lett. 78, 285 (2001). 